Article

\title{
Nested One-to-One Symmetric Classification Method on a Fuzzy SVM for Moving Vehicles
}

\author{
Guofeng Qin ${ }^{1, *}$, Xiaodi Huang ${ }^{2}$ and Yiling Chen ${ }^{1}$ \\ 1 The Department of Computer Science and Technology, Tongji University, Shanghai 200092, China; \\ 1631716@tongji.edu.cn \\ 2 School of Computing and Mathematics, Charles Sturt University, Albury, NSW 2640, Australia; \\ xhuang@csu.edu.au \\ * Correspondence: gfqing@aliyun.com; Tel.: +86-21-6598-1423
}

Academic Editor: Yuhua Luo

Received: 13 December 2016; Accepted: 23 March 2017; Published: 26 March 2017

\begin{abstract}
As a multi-classification problem, classification of moving vehicles has been studied by different statistical methods. These practical applications have various requirements, efficiencies, and performance, such as the size of training sample sets, convergence rate, and inseparable or ambiguous classification issues. With a reduction in its training time, the one-to-many support vector machine (SVM) method has an advantage over the standard SVM method by directly converting the binary classification problem into two multi-classification problems with short time and fast speed. When the number of training samples of a certain type is far less than the total number of samples, the accuracy of training, however, will be significantly decreased, leading to the problem of inseparable area. In this paper, the proposed nested one-to-one symmetric classification method on a fuzzy SVM symmetrically transforms the $C$ multi-classification problems into the $C(C-1) / 2$ binary classification problems with $\mathrm{C}(\mathrm{C}-1) / 2$ classifiers, and solves the problem of inseparable area. According to the best combination factor of kernel function $(\gamma, \mathrm{C})$ for the radial basis function (RBF) in the comparative experiments of training sample sets among the different algorithms, and the experimental results of many different training sample sets and test samples, the nested one-to-one symmetric classification algorithm on a fuzzy SVM for moving vehicle is able to obtain the best accuracy of recognition.
\end{abstract}

Keywords: support vector machine (SVM); parameter of RBF kernel; combination factor; mobile vehicle classification.

\section{Introduction}

Vehicle type classification is the key factor for diversion and guidance of modern intelligent traffic. It is necessary to overcome impacts on the quality and illumination of a video image because of the fast motion of vehicles, weather, and other factors. Vehicle classification normally consists of three steps. First, a target detection algorithm that detects vehicle images from video streams is designed, the features of vehicles from the detected images are then extracted and, finally, a classification classifier will identify the vehicles [1,2]. In particular, video image classification needs to overcome the disadvantages of piezoelectric detectors, infrared detectors, magnetic induction detectors [3], and ultrasonic detectors. Traditional vehicle classifiers are vulnerable to external instabilities with low accuracy [4]. The multi-classification process of vehicles that attempts to overcome these drawbacks is, therefore, introduced, which is described as follows: the first step is to obtain a known sample set with a known category; the second step is to train a classifier by a set of the known samples to refine the parameters of the objective judgment expression of the classifier; and the final step is to test the trained classifier against unclassified samples. In fact, the acquired samples often contain noise. It is 
easy for a classifier to mistaken on-classification objects as isolated or ambiguous points. This becomes a key indicator of measuring whether a classifier is accurate or not. In addition, the rapid convergence of identification and classification is also a requirement of classifying vehicles in real-time. Studies on these basic questions are helpful to quickly shunt and guide traffic flows in modern intelligent transportation $[5,6]$.

The essence of these questions is how to classify the data with high-dimensional features, which is studied in data mining, machine learning, and other fields [7-12]. In fact, many such algorithms are available [13-16], for example, nearest neighbor, naive Bayes classification algorithm, neural networks, support vector machines, cascade classifiers, and so on. Since Cover and Hart proposed the nearest neighbor method in 1968, K-nearest neighbor and nearest neighbor methods have extensively been studied and widely used [1]. These methods can be simplified through linear scanning, and require a large amount of training data. When the dimension of data features is high, the amount of calculations will be too heavy. This will need cost-intensive computation resources to search for neighboring samples, and consume a large amount of memory. It is also difficult to choose a distance function because the result of classification depends heavily on the parameters. As a relatively quick and mature recognition and classification algorithm, the BP neural network method is simple and easily implemented. However, a BP neural network is a heuristics approach with several disadvantages: the optimization process of BP algorithms, which is derived from the gradient descent, easily falls into a local minimum, so the coverage ability of neural network training may be weak. The BP neural network classification aims to minimize the training error with respect to the limited number of training samples, so the generalization of the BP algorithm is very weak. The structure of a neural network depends on experience knowledge because of lacks in rigorous theoretical support $[17,18]$. It is difficult to analyze, quantitatively, whether the process of neural network training converges or not. It cannot know the determinants of the convergence rate. Therefore, it is unable to determine whether the process is converging and obtain the speed of convergence. There exists uncertainty in the process of solving problems.

The research team from AT\&T Bell Laboratories led by Vapnik proposed the classification method of the support vector machine (SVM) that overcomes the inherent defects of the BP network and is better suited to handle the convergence rate of vehicle classification and the certainty of solving classification problems $[19,20]$. The method has three advantages: it obtains an optimal solution under a priori information with a limited number of samples; it converts the classification problem into a question two times for searching the excellent classification and obtaining the global optimal solution; and it converts data into a high dimensional feature space by non-linear transformation, which has a good generalize ability, and solves the computation resource problem because of the high dimensionality [21,22]. The basic idea of the SVM is to find an optimal classification hyper-plane that must meet the classification purposes, which not only ensures the classification accuracy, but also maximizes the blank area on both sides of the hyper-plane. An SVM can achieve optimal classification for linearly separable problems, and convert a nonlinear problem into a problem of linear, high-dimensional space with the method of nuclear mapping [23,24]. At first, the SVM is mainly used to solve the problem of binary classification. Of course, most of the practical classification problems frequently encountered should usually be classified into two types $[25,26]$. However, the classification of vehicles is a multi-classification problem. Thus, the basic support vector machine algorithm should be improved to solve the practical multi-classification problem [27]. No matter whether an algorithm is for one-to-one or one-to-many classification, there always exist regions in an image that cannot be classified by the algorithm. In order to solve this problem, Abe introduced a decision function based on fuzzy support vector machines [28-30]. Platt, et al. presented a directed acyclic graph based on the decision tree. Kijsirikul, et al. proposed an adaptive directed acyclic graph [31]. Cheong and Fei [32] proposed a binary tree algorithm, which solves the problem of inseparable regions with coarse granularity, but it will introduce accuracy problems in classification. After comparing different performances between multi-classification algorithms, Hsu et al. [33] made some important evaluations of the optimization 
with the time-domain and space-domain. The classification of the moving vehicle is regarded as a multi-classification problem [34]. The accurate classification statistics of different types is always the basis requirement of traffic management and the decision-making in real-time by traffic officers. The size of training samples, the convergence rate of solving the classification problem, and inseparable regions or ambiguous classifications are the main influencing factors of multi-classifications methods. All of these facts motivate us to use the fuzzy support vector machine, to study the algorithms of the nested one-to-one symmetric classifier model, and to construct multi-classification classifiers for moving vehicles in the video domain. The algorithm can combine the advantages of a fuzzy judgment function and a support vector machine, measure the contribution of each sample point more accurately, handle the metric problem of the classified sample properly, and solve the regression of samples with noise rather well.

The organization of this paper is as follows: In Section 2, we will propose a nested one-to-one symmetric classification algorithm using a fuzzy support vector machine (FSVM), based on of the analysis of a one-to-many SVM and directed acyclic graph multi-classification algorithm. The algorithm is verified by classification experiments on moving vehicles. The algorithm symmetrically transforms $\mathrm{C}$ multi-classifications into $C(C-1) / 2$ two-classifications with $C(C-1) / 2$ classifiers, and solves the problem of inseparability areas. Section 3 reports the experimental results. The same sample set and the same test set are used to compare the results of the nested one-to-one symmetric classification algorithm on the FSVM with other algorithms to verify the strong stability and accuracy of the proposed algorithm. Section 4 concludes this paper.

\section{Construction of the Model of a Nested One-to-One Symmetric Classification Classifier on FSVM}

\subsection{The Basis of the Multi-Classifier Algorithm}

The one-to-many support vector machine classification algorithm that Vapnik proposed is the first multi-classification algorithm. Its basic idea is to construct multiple standards for a support vector machine classifier that can classify two categories. Each classifier, respectively, uses the discrete discrimination function to separate one category from all of the samples by the SVM binary classification algorithm. In the testing process, the testing samples of unknown classes are individually input into the classifier to decide its class, and taking the category which has the maximum of the decision function as the final class which the test sample belongs to. Many SVM methods are multi-classification algorithms with their simple and easy operations. Their advantage is that the standard support vector machine method can be directly used to convert the multi-classification problem into two binary classification problems. For example, a classification problem with $\mathrm{C}$ classes can be converted to a two-classification problem with $\mathrm{C}$. It just needs $\mathrm{C}$ two-category classifiers to train the set of samples, so the training time is much shorter and faster. When the number of training samples of a certain type are far less than the total number of samples, the accuracy of training will be extremely decreased because of the problem of inseparable regions. These limitations still exist in the one-to-many support vector machine classification algorithm.

In order to solve the inseparable regional issues in one to many support vector machine multi-classification, Platt et al. presented a tree-based directed acyclic graph support vector machine (DDAG SVM) in 2000. The DDAG tree of three classification problems is shown in Figure 1, $\bar{m}$ represents that sample $x$ does not belongs to the $m$ class, while the top-level classifier can constitute any two binary classification classifiers. In addition to the leaf node, if the decision function $D_{m n}(x)>0$, $x$ does not belong to the category of the $n$ class, or $x$ do not belong to the $m$ class. The height of the C-categories DDAG tree is C minus 1, namely C -1 .

A directed acyclic graph is a standard combination algorithm that combines SVM and binary classification classifiers without searching for all categories. It has high efficiency and accuracy. However, the different methods of generating DDAG trees will result in assigning an inseparable region to different leaf nodes. Thus, the algorithm depends heavily on the structure of the decision 
tree. Another major drawback of this method is that once the root node is chosen incorrectly, it will lead to subsequent misclassification. In this sense, the algorithm is unstable. In addition, the number of binary classification classifiers will increase as the number of classification categories increases. The uncertainty and certainty of the classification algorithm become the foundation and motivation for us to improve the algorithm.

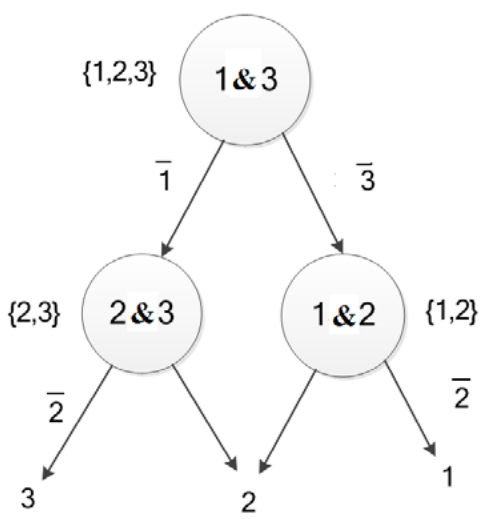

Figure 1. DDAG tree of the three classification problem.

\subsection{The Model of the Nested One-to-One Symmetric Classification on a Fuzzy Support Vector Machine}

In the real situation of vehicle classification, the collected samples often contain noise or isolated points. A fuzzy judgment function on the degree of membership is a better solution to solve the sensitive issues of outliers or noises in SVM and more appropriate to the metric scope of the class which the sample belongs to. By combining the two advantages, a nested one-to-one symmetric classification algorithm based on a fuzzy support vector is proposed, which not only measures the contribution of each sample point more accurately, but also solves the regression problem of samples with noise well.

Assuming there are the number of $l$ training samples $x_{1}, x_{2}, \ldots, x_{1}$ with L dimensions, they fall into two categories, $\mathrm{H}_{1}$ and $\mathrm{H}_{2}$. The attribute value for each training sample is $y_{i}$, with $y_{i}=+1$ as a representation of $\mathrm{x}_{\mathrm{i}}$ in category $\mathrm{H}_{1}$, and $y_{i}=-1$ as a representation of $x_{i}$ in category $\mathrm{H}_{2}$. The sample sets is expressed as:

$$
\left(x_{1}, y_{1}\right),\left(x_{2}, y_{2}\right), \ldots,\left(x_{l}, y_{l}\right), x_{i} \in R^{L}, y \in\{-1,+1\}
$$

Continuing to blur it as follows:

$$
\left(x_{1}, y_{1}, s_{1}\right),\left(x_{2}, y_{2}, s_{2}\right), \ldots,\left(x_{l}, y_{l}, s_{l}\right)
$$

In Equation (2), $s_{i}$ represents the fuzzy degree of membership, representing the degree value $x_{i}$ that belongs to a particular class, and satisfies $\sigma \leq s_{i} \leq 1, i=1,2, \ldots, l$, for a sufficiently small constant $\sigma>0$. In this case, the optimal problem based on the fuzzy support vector machine can be changed as follows:

$$
\min \frac{1}{2}\|w\|^{2}+C \sum_{i=1}^{l}\left(s_{i} \xi_{i}\right)
$$

Equation (3) is subject to the following condition:

$$
\left\{\begin{array}{c}
y_{i}\left(w \bullet \varphi\left(x_{i}\right)+b\right) \geq 1-\xi_{i} \\
\xi_{i} \geq 0
\end{array}, i=1,2, \ldots, l\right.
$$

In Equation (3), $w$ is the weight of a linear discriminant function, $w \bullet \phi\left(x_{i}\right)$ is the inner product of vector $w$ and $\phi\left(x_{i}\right), \mathrm{b}$ is a hyper-plane deviation constant, and $\xi_{i}$ is a slack variable. 
The correspondent Lagrangian formula is as follows:

$$
\begin{aligned}
& \mathrm{L}(w, b, \xi, \alpha, \beta) \\
& =\frac{1}{2}\|w\|^{2}+C \sum_{i=1}^{l}\left(s_{i} \xi_{i}\right)-\sum_{i=1}^{l} \alpha_{i}\left(y_{i}\left(w \cdot \phi\left(x_{i}\right)+b\right)-1+\xi_{i}\right)-\sum_{i=1}^{l} \beta_{i} \xi_{i}
\end{aligned}
$$

Solving the saddle point $\mathrm{L}(w, b, \xi, \alpha, \beta)$ the parameters must meet the following condition:

$$
\left\{\begin{array}{c}
\frac{\partial L(w, b, \xi, \xi, \alpha, \beta)}{\partial w}=w-\sum_{i=1}^{l} \alpha_{i} y_{i} \phi\left(x_{i}\right)=0 \\
\frac{\partial L(w, b, \xi, \alpha, \beta)}{\partial b}=-\sum_{i=1}^{l} \alpha_{i} y_{i}=0 \\
\frac{\partial L(w, b, \xi, \alpha, \beta)}{\partial \xi_{i}}=s_{i} C-\alpha_{i}-\beta_{i}=0
\end{array}\right.
$$

Equation (4) is substituted into Equation (5), and is optimally solved as follows:

$$
\max _{\alpha} w(\alpha)=\max \left\{\sum_{i=1}^{l} \alpha_{i}-\frac{1}{2} \sum_{i=1}^{l} \sum_{j=1}^{l} \alpha_{i} \alpha_{j} y_{i} y_{j} K\left(x_{i}, x_{j}\right)\right\}
$$

In Equation (6), $K\left(x_{i}, x_{j}\right)=\phi\left(x_{i}\right) \bullet \phi\left(x_{j}\right)$ is a symmetric function (named as a kernel function). It meets the following constraint criteria:

$$
\left\{\begin{array}{l}
\sum_{i=1}^{l} \alpha_{i} y_{i}=0 \\
0 \leq \alpha_{i} \leq s_{i} C
\end{array}, i=1,2, \ldots, l\right.
$$

For the bipartition question, the value of $s_{i}$ can be determined by the center of the class. Let the means of class +1 and -1 be, respectively, $x_{+}$and $x_{-}$; the radius of sample type +1 is as follows:

$$
r_{+}=\max _{x_{i}: y=1}\left|x_{+}-x_{i}\right|
$$

The radius of the sample type -1 is as follows:

$$
r_{-}=\max _{x_{i}: y=-1}\left|x_{-}-x_{i}\right|
$$

$s_{i}$ is valued as follows:

$$
S_{i}=\left\{\begin{array}{c}
1-\left|x_{+}-x_{i}\right| /\left(r_{+}+\delta\right), \text { if } y_{i}=1 \\
1-\left|x_{-}-x_{i}\right| /\left(r_{-}+\delta\right), \text { if } y_{i}=-1
\end{array}\right.
$$

where, $\delta>0$ and $s_{i} \neq 0$.

The one-to-many algorithm and the directed acyclic graph multi-classification algorithm still could not completely resolve the inseparable regional issues of SVM. In this paper, a nested algorithm based on a one-to-one symmetric strategy is proposed to handle the multi-classification problem. In the following we will theoretically prove the high convergence of the nested one-to-one symmetric classification by decreasing the number of samples in the inseparable regions to just one or two classes, or even no sample.

The nested one-to-one symmetric classification algorithm converts a C-categories classification into a problem of $\mathrm{C}(\mathrm{C}-1) / 2$ binary classification classifiers. The $m$-th classifier classifies the samples that belong to the $m$-th class into a positive class, and the rest of the samples into a negative class. A hyper-plane is between the $m$-th class and the $n$-th class as follows:

$$
D_{m n}(x)=w_{m n}^{T} \phi(x)+b_{m n}
$$




$$
D_{m n}(x)=-D_{n m}(x)
$$

In Equation (10) $w_{m n}$ is the weight matrix of a linear discriminant function between the $m$-th class and the $n$-th class, and $b_{m n}$ is an interval distance constant between the $m$-th class and the $n$-th class. The discrete discrimination function for a given sample $x$ is as follows:

$$
D_{m}(x)=\sum_{n \neq m, n=1}^{C} \operatorname{sgn}\left(D_{m n}(x)\right)
$$

where:

$$
\operatorname{sgn}\left(D_{m n}(x)\right)= \begin{cases}1, & D_{m n}(x) \geq 0 \\ 0, & D_{m n}(x)<0\end{cases}
$$

In Equations (12) and (13), C is times of C-categories classification.

If a sample $x$ meets:

$$
k=\underset{m=1, \ldots, C}{\operatorname{argmax}}\left(D_{m}(x)\right)
$$

Then the sample $\mathrm{x}$ is classified as the $k$-th class. If multiple values of $k$ are equal, then the sample $x$ is inseparable. Under normal circumstances, the algorithm results in the inseparable regions having a value of 6 , such as the three-classification problem shown in Figure 2.

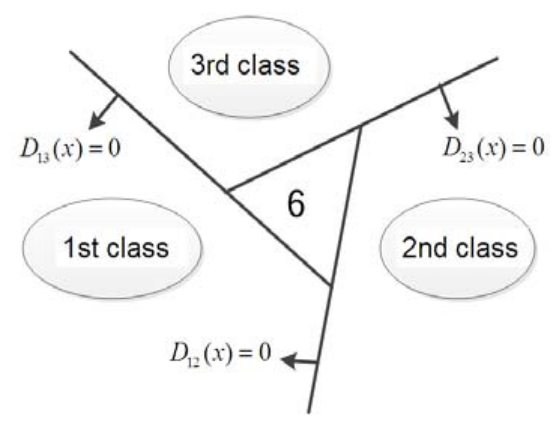

Figure 2. The result of the nested one-to-one algorithm.

The steps of the nested one-to-one symmetric classification algorithm are detailed as follows:

(1) For a $\mathrm{C}$ classification problem, the nested one to one classification constructs the most superior $\mathrm{C}(\mathrm{C}-1) / 2$ hyper-planes. Where the hyper-plane between the $m$-th class and the $n$-th class is as Equation (10), with $\mathrm{m}$ and $n \in\{1,2,3\}$. A three-classification of the vehicle classification problem is addressed in this work, and three optimal hyper-planes are established.

(2) For any given sample $x$, if the result $k$ is the only value by calculating according to Equation (13), then sample $\mathrm{x}$ belongs to the $k$-th category. Otherwise, $x$ will fall into the samples of the inseparable region according to Equation (13).

(3) If the number of categories of samples that fall into the category of the non-separable area is greater than, or equal to, 3 , then it is a multi-classification problem. Using these samples constructs three hyper-planes according to the symmetric strategy of the nested one-to-one classification.

(4) Repeat steps (2) to (3) until the inseparable area contains only one or two classes, or no sample.

(5) If the inseparable area contains only one type of sample, finally, the area is assigned to one class; if it contains two types of samples, the binary classification fuzzy support vector machine would be used to divide the region and assign the corresponding category. 


\subsection{Implementation and Validation of the Nested One-to-One Symmetric Classification Model on FSVM}

In order to validate the nested one to one symmetric classification model based on FSVM, traffic streams in different scenarios are used to successfully realize multi-classification for different type of vehicles in this work. Experimental samples are divided primarily into three categories, namely cars (including jeeps, cars), vans (including ordinary vans and commercial vehicles), and buses, being represented by their capital letters C, V, and B. The experiments extracted more than 2000 key frames from the different video streams in order to construct a sample library of vehicle images. Six types of cars, vans, and buses had been collected, with 10 samples of each type on the front of the vehicles in non-sunny, cloudy, rain, snow, and night. The numbers of sample libraries of vehicle images are listed in Table 1, with the vehicle types shown in Figure 3.
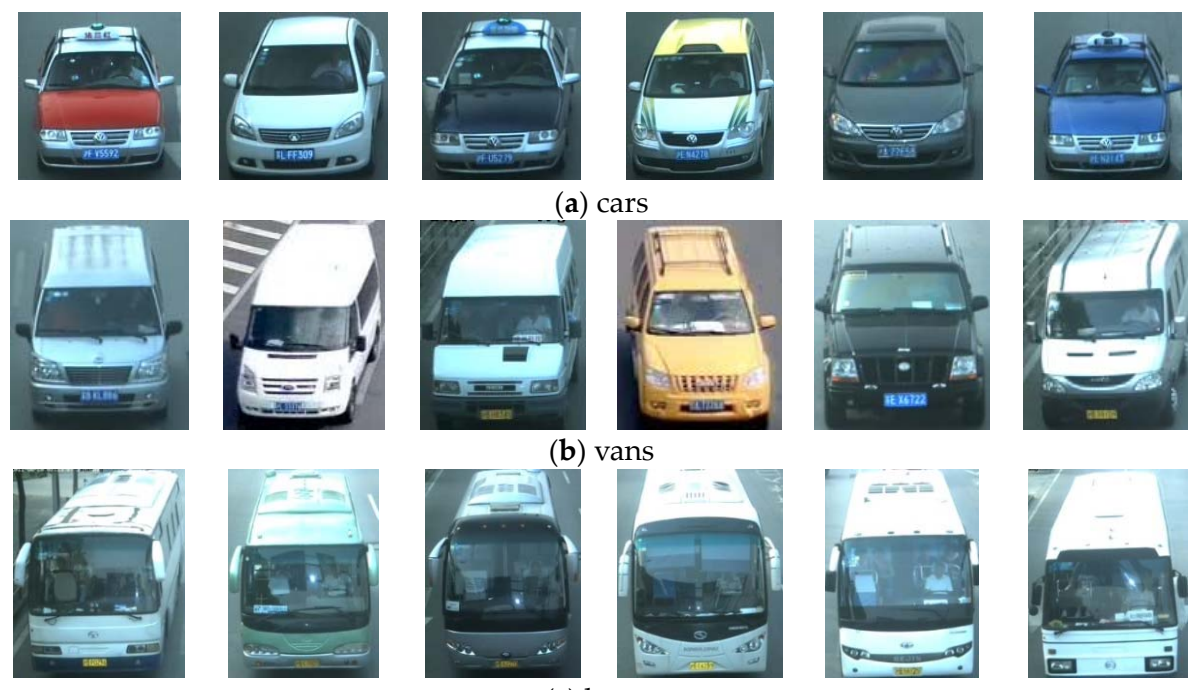

(a) cars
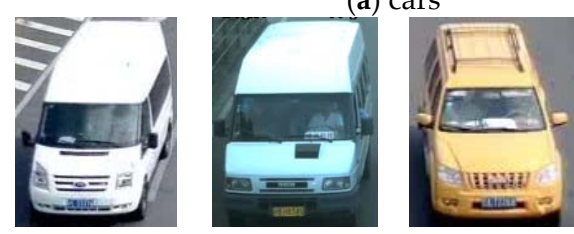

(b) vans

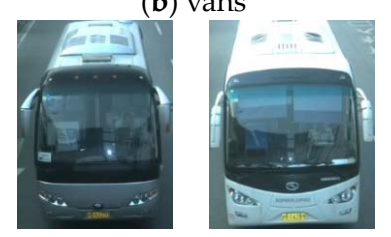

(c) buses
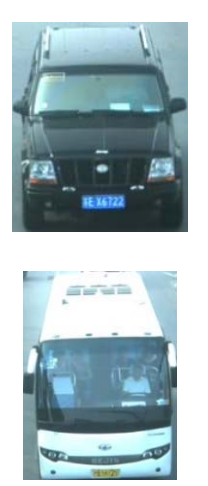
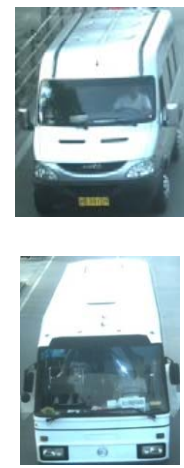

Figure 3. Vehicle images of the sample library.

Table 1. The numbers of sample libraries of vehicle images.

\begin{tabular}{cccc}
\hline No. & Name & Number & Video Capture Direction \\
\hline 1 & Car & 60 & front \\
2 & Van & 60 & front \\
3 & Bus & 60 & front \\
\hline
\end{tabular}

In order to validate the accuracy of the algorithm, our first work is to pre-process the video image, including video analysis which separates the background and the target vehicle, the extraction of the target vehicles through a virtual centroid reference line in the key frame, and the extraction of five dimensional feature vectors of vehicle characteristics. Feature information of the vehicle classification input source is cleaned, and then we improve the vehicle classification algorithm with comparative analysis of experiments. The method details are as follows:

(1) The detection of vehicle based on the analysis of video.

At first, the images from the captured stream of video are pre-processed, and the block mixture Gaussian algorithm, which is a fused Bayesian decision theory, is used to detect vehicles. The procedure includes three steps. It starts with establishing the model of the background by using the block mixture Gaussian algorithm in order to obtain a probability distribution function for each pixel in the image frame from the video stream; the second step is to extract the foreground objects in the video and obtain a connected prospects area by forward correlation with Bayesian decision theory; the third step is to determine whether the foreground area is derived from the target vehicle or noise through 
connectivity analysis, which separates the extracted target vehicle from the background. Meanwhile, the background of images is updated by the improved block mixture Gaussian model.

(2) Extraction of key frames.

For each frame image in a video stream, an artificially-fixed virtual line is chosen to pick the frame image whose virtual line is through the vehicle centroid as key frames. After the target vehicles are extracted by the vehicle detection algorithm, edge detections and morphological operations will be conducted. The processed key frames are then segmented and formalized to standardization images with the size of $90 \times 60$ pixels.

(3) Extraction of features of target image.

Firstly, the shape and texture features of the vehicle's image are extracted, including scale-invariant feature transform (SIFT) features of vehicles images, the dimensions of which are decreased by a PCA algorithm. Finally, the linear discrimination analysis of the combination of vehicle feature vectors is used to determine the best class.

A seven-dimensional shape feature vector consists of the seven invariant moments normalized $F=\left(f_{1}, f_{2}, f_{3}, f_{4}, f_{5}, f_{6}, f_{7}\right)$. A five vehicle-dimensional texture feature vector consists of the mean, standard deviation, the number of pixels of regions, third moment, and entropy $V=\left(m, \sigma, S, \mu_{3}, e\right)$.

In order to enhance the stability and the ability of anti-noise of characteristic points, a neighborhood window of $16 \times 16$ key points is selected to extract the SIFT features of an image. Each $16 \times 16$ window will be segmented into 16 sub-windows of $4 \times 4$, with each child window generating eight directions of seeds. Thus, each key has a total of $16 \times 8=128$ dimension descriptors of SIFT features. The SIFT feature vectors of all target vehicle images are stacked into a matrix and reduced into a 19-dimension vector by the PCA algorithm.

A 19-dimensional SIFT feature vector concatenates the $7+5=12$ dimension shape and texture feature vectors to form a $19+12=31$ dimension feature vector of a vehicle. The class scatter matrix $S_{w}$ and class scatter matrix $S_{b}$ of the space of 31 intra-dimensional features of the vehicle are calculated by the linear discriminant analysis (LDA) algorithm, and the first five eigenvectors of $S_{w}^{-1} S_{b}$ are taken as the projection matrix of the space of LDA features. Therefore, the features of a vehicle are represented by the final five-dimensional feature vector.

(4) The selection of the kernel function and determination of parameter of the fuzzy support vector machine.

The kernel function is the key factor in affecting the accuracy rate of classification in multi-classification by an SVM. Since the radial basis kernel function is a non-linear mapping of the kernel function, which is good at dealing with the nonlinear separable problem, the radial basis kernel function $K\left(x_{i}, x\right)=\exp \left(-\gamma\left\|x_{i}-x\right\|_{2}\right)$ parameters $\gamma \in\left[2^{-10}, 2^{2}\right]$ are selected, where $x_{i}$ is a sample point, and $\mathrm{x}$ is a center point of kernel function. Vapnik et al. have demonstrated that nuclear parameters $\gamma$ and error penalty factor C are key factors affecting the performance of the SVM [27]. The effect on SVM is related to the kernel parameters $\gamma$ and penalty factor $C$. The relevant curve between the error rate and penalty factor $C$ is a declivity curve when kernel parameter $\gamma$ is set as a constant. For example, when the penalty factor $\lg (C) \in[-8,-5]$, the sub-curve is a flat line with an error rate $=70 \%$; when $\lg (C) \in(-5,-1]$, the sub-curve is a descending parabolic curve with an error rate $\in(70 \%, 10 \%]$, when $\lg (C) \in(-1,2]$, the sub-curve also is a flat line with an error rate $=10 \%$. On the other hand, the relevant curve between the error rate and penalty factor $\mathrm{C}$ is a V-shaped sharp curve between the error rate and the kernel parameters $\gamma$ when the penalty factor $\mathrm{C}$ is set as a constant. For example, when the kernel parameters $\lg (\gamma) \in[-8,-6]$, the sub-curve is a diagonal line with the error rate $\in[75 \%, 70 \%]$; when $\lg (\gamma) \in(-6,0.5]$, the sub-curve is a symmetric parabolic curve with the error rate $\in[10 \%, 70 \%]$; and when $\lg (\gamma) \in(0.5,2]$, the sub-curve is a diagonal line with the error rate $\in(70 \%, 65 \%]$. 
A testing set with 1500 samples is used to train. The values of $C$ and $\gamma$ are adjusted to test the relationship with the accuracy of the recognition and classification. The relationship curves can be seen in Figure 4. The three-dimensional surface shows the error rate with continuous change affection with kernel parameters $\gamma$ and penalty factor $C$ in the experiment of the testing set. From the experiments, we have found the best combination of parameters $(\gamma, C)=(0.001,10)$ with the rate of correct classification being $92.47 \%$; namely, the error rate is $7.53 \%$. The comparisons of the accuracy of classification between other classification algorithms and this algorithm will be presented later.

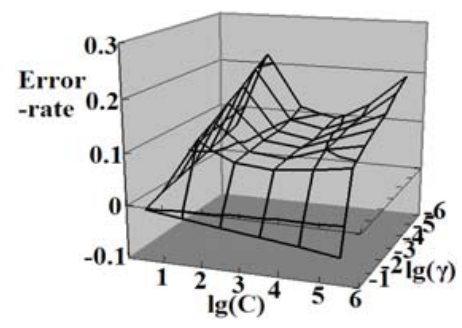

Figure 4. Error rate of classification on different combination $(C, \gamma)$.

(5) The design of the nested one to one symmetric classifier.

How to design a symmetric classifier based on FSVM is the most critical part of the entire test system. For the problem of three vehicle type classification, we first construct three $(3 \times(3-1) / 2)$ nested one-to-one symmetric classifiers, which are based on the symmetric strategy, namely, $(\mathrm{B}, \mathrm{C}+\mathrm{V})$, $(C, B+V)$, and $(V, B+C)$. The $(B, C+V)$ classifier indicates that the buses are divided into a positive category, while the cars and vans are divided into a negative category, and so on. Then, the nested one-to-one symmetric classification algorithm is used to classify the types of vehicles.

The process of the nested one-to-one symmetric classification algorithm for moving vehicles based on FSVM is illustrated in Figure 5.

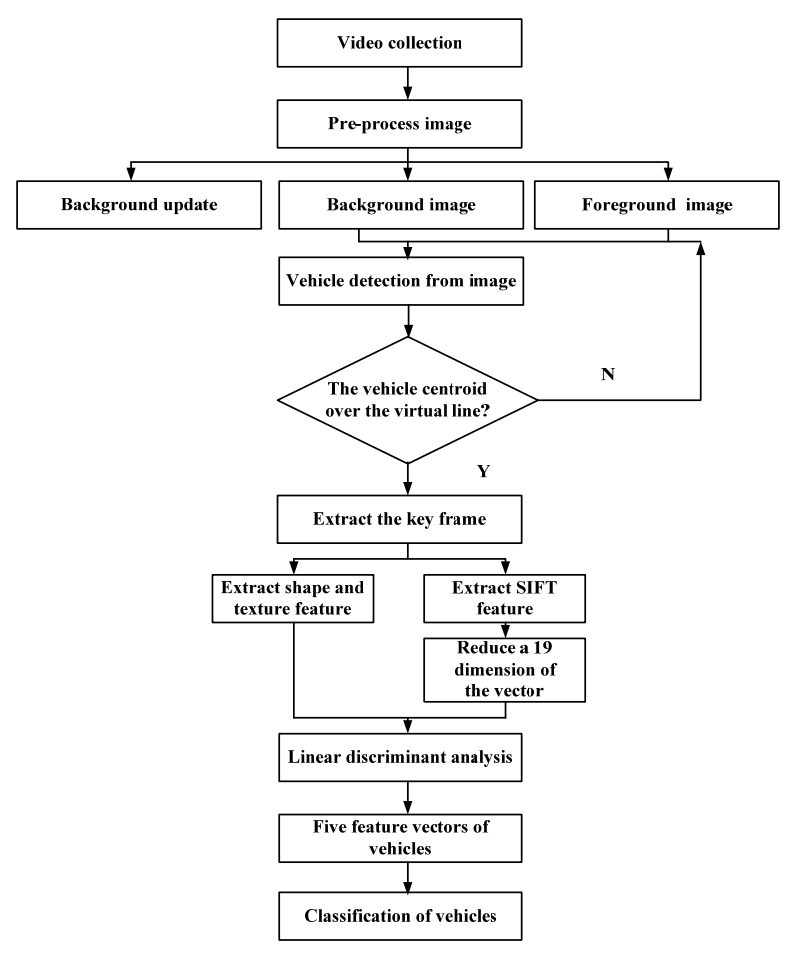

Figure 5. The process of the nested one-to-one symmetric classification algorithm based on FSVM for moving vehicles. 


\section{Experiment of the Nested One-to-One Symmetric Classification for Moving Vehicles Based on FSVM}

\subsection{Procedure of Experiment}

In the simulation of experiment, interface of a computer vision library in OpenCV (Intel, Santa Clara, CA, USA) is used, and a classification system of moving vehicles in the Visual Studio 2010 (Microsoft, Redmond, Washington, DC, USA) is developed. OpenCV supplies a cross-platform API with more than $500 \mathrm{C}$ functions, does not depend on external libraries, and has many rich visual processing algorithms. Therefore, OpenCV is a good choice for conducting experiments of vehicle recognition based on video analysis. The videos in the experiment are two videos of traffic streams under different scenarios over $45 \mathrm{~min}$. In particular, scene 1 has people and vehicles in the streets, as shown in Figure 6, while scene 2 is of peak time on the highway, as shown in Figure 7.

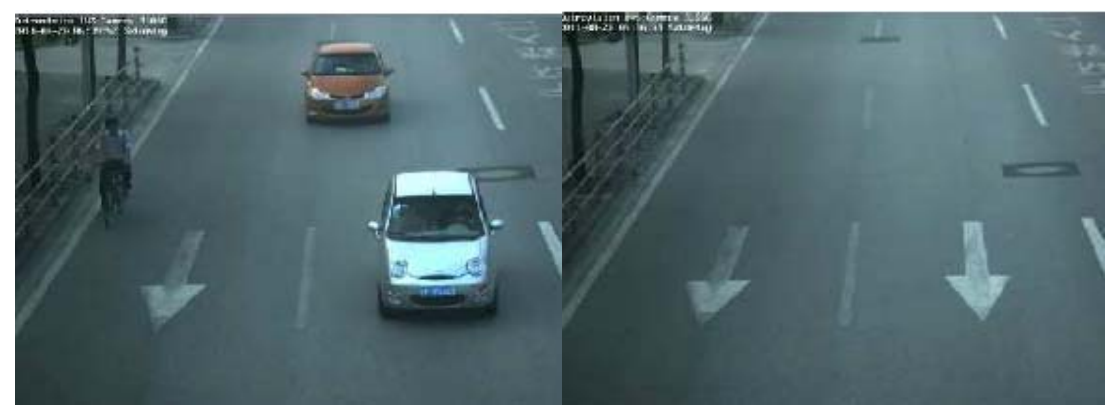

Figure 6. An original picture of scene 1 (left) and background (right).

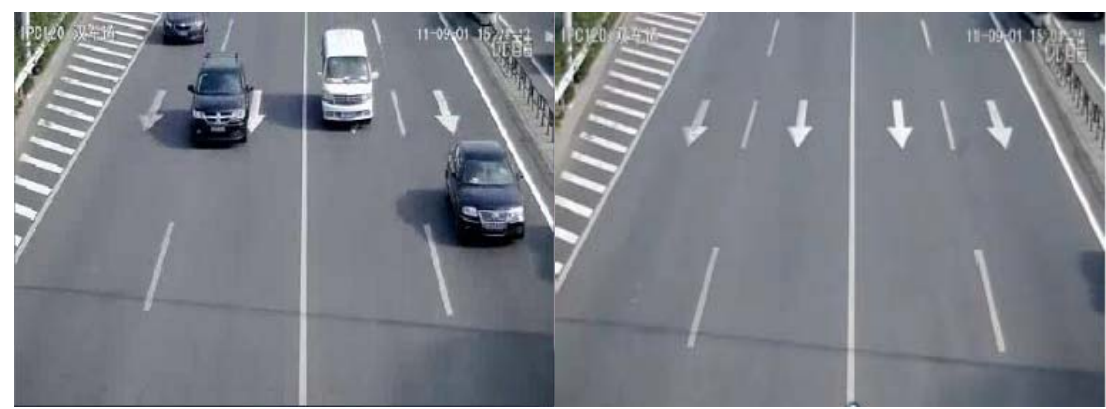

Figure 7. An original picture of scene 2 (left) and background (right).

(1) Simulations of vehicle testing.

In order to verify the performance of the improved algorithm of the Gaussian block texture for vehicle testing, we performed the comparative analysis by simulation. Experiments used statistical algorithms of traffic based on virtual coils as shown in Figure 8, the comparative analysis is undertaken with different vehicle testing algorithms, and the obtained results are reported in Table 2.

Table 2. Traffic statistics table.

\begin{tabular}{ccc}
\hline Recognition Rate (\%) & Scene 1 & Scene 2 \\
\hline Vehicle detection algorithm based on the classical Gaussian mixture model & 98.4 & 94.3 \\
Gaussian mixture algorithm based on Bayesian Block & 98.6 & 98.1 \\
\hline
\end{tabular}

In terms of identifying the movement of the vehicle, from Table 2, the improved Gaussian mixture algorithm based on Bayesian Block for vehicle detection has not improved the accuracy in a simple 
scenario significantly. With respect to Scenario 2, whose background is complicated by the distraction of shadows, the improved Gaussian mixture algorithm based on Bayesian Block can extract the features of the moving target vehicle more accurately than the traditional Gaussian mixture algorithm. The improved algorithm has the higher identification rate, and provides a good basis for classification detection algorithms at the following steps.

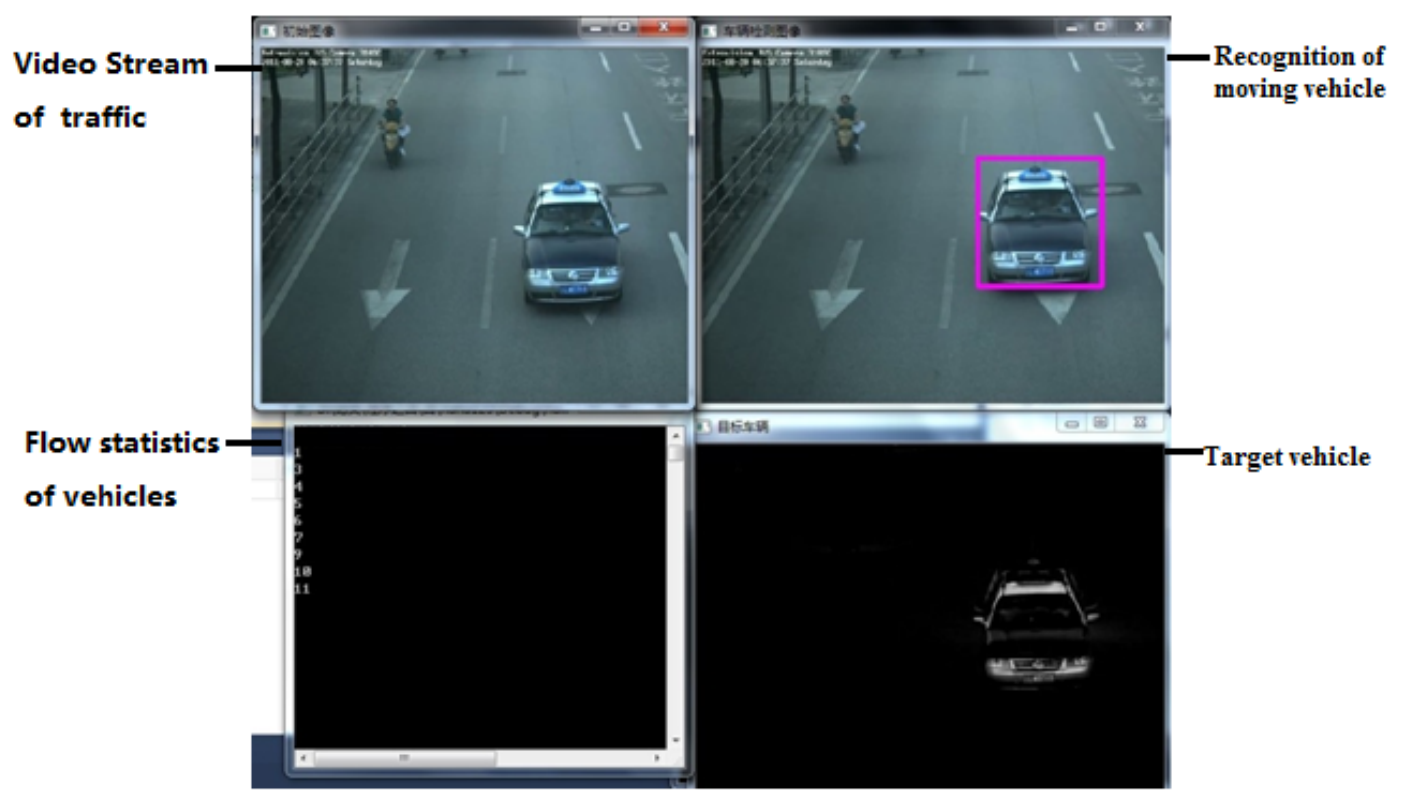

Figure 8. Renderings of an experimental run.

(2) The experiments of feature extraction.

For verifying the superiority of the reduced SIFT feature dimension with vehicle characteristics of vehicle shape and texture, a comparative experiment has been conducted, namely, a comparative test was made between the experiment with shape and texture features of the vehicle, and with the combination of their features and reduced SIFT dimension. The linear discrimination analysis algorithm is used to process the feature vector. The final results are compared in Figure 9.
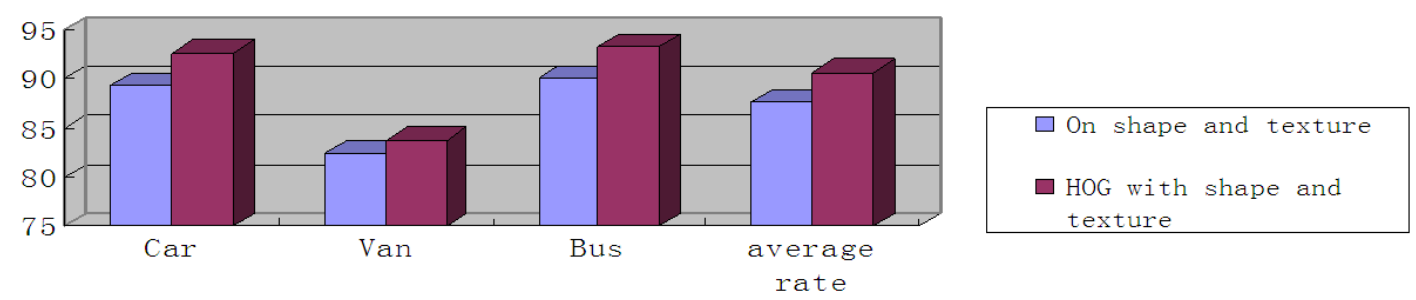

Figure 9. The correct rate (\%) of identification of characteristics of different vehicles.

In Figure 9, the combination of SIFT features, shape and texture features were used to describe the vehicle characteristics. This method can greatly improve the accuracy of recognition models. The feasibility and effectiveness of the improved feature extraction methods are, thereby, verified.

The adequacy and effectiveness of the recognition of moving vehicles and the extraction of vehicle features are the prerequisites for testing multi-classification algorithms of vehicle models.

(3) Compared experiments on the algorithms of multi-classification.

In order to validate the effectiveness of the proposed nested one-to-one symmetric classification algorithm, we conduct a comparative analysis among the one-to-many classification algorithm, directed 
acyclic graph classification algorithm, and the nested one-to-one symmetric classification algorithm against different training sets and testing sets of images by experiment. The experimental testing software interface is shown in Figure 10. When the video stream of traffic from the monitors is shown on the right window, the software of the nested one-to-one symmetric classification algorithm on the fuzzy SVM will be run to classify the moving vehicles. The results of the classification sequence are displayed in the left window. At the same time, the classification data will be added to the database of the traffic management system in real-time.

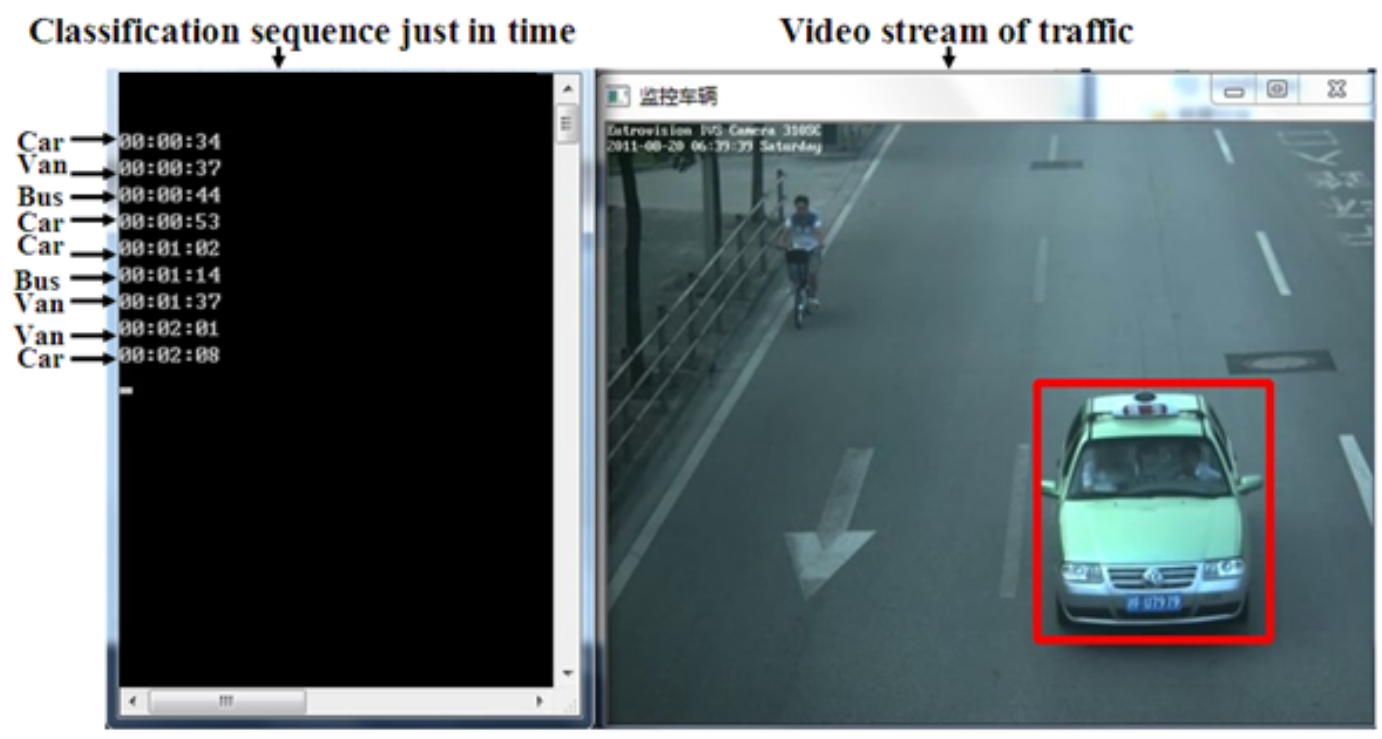

Figure 10. Software interface of moving vehicle classification.

\subsection{Analysis of Results}

For verifying the algorithm of the vehicle classifier, the experimental datasets are selected from the vehicle image library, where the number of samples are, respectively, 600, 900, 1200, 1500, 1800, and 2100. Two thirds of the sample images are used as the training sample set, and the remaining $1 / 3$ as the testing set. The experimental testing software interface is seen in Figure 8 . The classification results are compared against training sets and testing sets among directed acyclic graph classification, one-to-many classification, and the nested one-to-one symmetric classification. The accuracy rates of classifying the images in the training set are reported in Table 3, and the accuracy rates of classifying the images in the testing set was reported in Table 4.

Table 3. Comparisons of accuracy of the training sets.

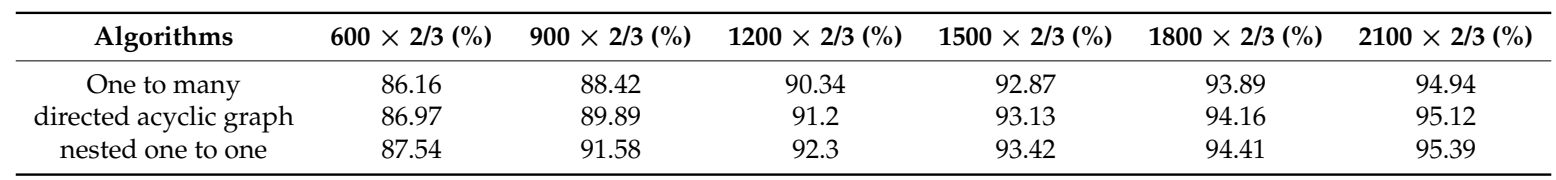

Table 4. Comparisons of accuracy of the testing sets.

\begin{tabular}{ccccccc}
\hline Algorithms & $\mathbf{6 0 0} \times \mathbf{1 / 3} \mathbf{( \% )}$ & $\mathbf{9 0 0} \times \mathbf{1 / 3} \mathbf{( \% )}$ & $\mathbf{1 2 0 0} \times \mathbf{1 / 3} \mathbf{( \% )}$ & $\mathbf{1 5 0 0} \times \mathbf{1 / 3} \mathbf{( \% )}$ & $\mathbf{1 8 0 0} \times \mathbf{1 / 3} \mathbf{( \% )}$ & $\mathbf{2 1 0 0} \times \mathbf{1 / 3} \mathbf{( \% )}$ \\
\hline One to many & 84 & 86 & 88.45 & 92.1 & 93.17 & 94.14 \\
directed acyclic graph & 84.4 & 87.33 & 89.1 & 92.18 & 93.26 & 94.22 \\
nested one to one & 85 & 88 & 90.12 & 92.47 & 93.48 & 94.43 \\
\hline
\end{tabular}

The accuracy curves of the different algorithms of vehicle classification are shown in Figure 11. The results show that the nested one-to-one symmetric classification algorithm based on FSVM has 
the higher accuracy than both one-to-many classification and directed acyclic graph classification. This reason is that the accuracy of the one-to-many classification algorithm will be significantly decreased as the number of training samples is far less than the total number of samples, and it does not solve the problem of inseparable regions. On the other hand, if the root node is chosen incorrectly, the directed acyclic graph will lead to errors of classification with poor stability, and the number of two-classifiers will increase with the number of classification categories increasing in geometric progression. Therefore, the nested one-to-one symmetric classification algorithm on FSVM has strong stability and accuracy.

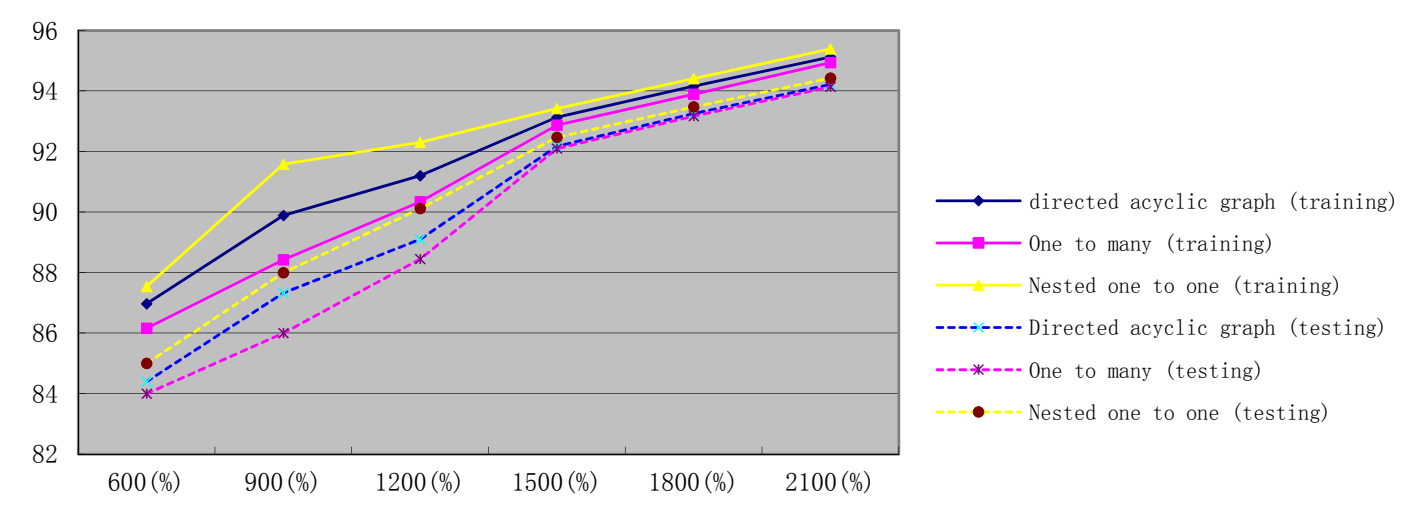

Figure 11. Classification accuracies of different algorithms between the training set and the testing set.

\section{Conclusions}

In this paper, we have presented a nested one-to-one symmetric classification method on FSVM for vehicle classification. In order to improve the classification accuracy of the algorithm, we improved several prior period methods by combining vehicle features in terms of the recognition of moving vehicles and the extraction of vehicle features. In particular, we improved the mixture Gaussian algorithm and the linear discrimination analysis method to purify the input feature information source of the symmetric classifier and enhance the quality of signals. These improvements help the nested one-to-one symmetric classification on the fuzzy SVM to classify vehicles into different classes and achieve an optimal statistical method of vehicle classification. By comparing among the classification algorithms, the experiment results have shown that the nested one-to-one symmetric classification algorithm on the fuzzy SVM is able to obtain the best accuracy of recognition. In the future, the real-time aspect of the proposed algorithm should be improved.

Acknowledgments: The researcher is supported by the national 863 program in Ministry of Science and Technology of the People's Republic of China. Project number: 2013AA040302.

Author Contributions: The correspondence author: Guofeng Qin works in the Department of Computer Science and Technology as an associate Professor, Tongji University, Shanghai, 201804, China, proposed the nested one-to-one symmetric classification algorithm. He received a BA from Hunan University in 1995, MA from Huazhong University of Science and Technology in 2001, and a PhD from Tongii University in 2004. Guofeng Qin proposed the nested one-to-one symmetric classification algorithm. Xiaodi Huang works at Charles Sturt University, Australia. Yiling Chen works in the Department of Computer Science and Technology as an MA student, Tongji University, Shanghai, 201804, China. Xiaodi Huang and Yiling Chen provided the test tools and environment for the nested one-to-one symmetric classification algorithm, and analyzed the test data. All of the authors wrote this paper.

Conflicts of Interest: The authors declare no conflict of interest.

\section{References}

1. Cover, T.; Hart, P. Nearest neighbor pattern classification. IEEE Trans. Inf. Theory 1967, 13, 21-27. [CrossRef]

2. Gupte, S.; Masoud, O.; Martin, R.F.K. Detection and Classification of Vehicles. IEEE Trans. Intell. Transp. Syst. 2002, 3, 37-47. [CrossRef] 
3. Gajda, J.; Sroka, R.; Stencel, M.; Wajda, A.; Zeglen, T. A vehicle recognition based on inductive loop detectors. In Proceedings of the IEEE Instrumentation and Measurement Technology Conference, Budapest, Hungary, 21-23 May 2001; pp. 460-464.

4. Michalski, A. Dry calibration procedure of electromagnetic flowmeter for open channels. IEEE Trans. Instrum. Meas. 2000, 49, 434-438. [CrossRef]

5. Merrier, R.; Harrington, A.; Cahill, V. A framework for integrating existing and novel Intelligent Transportation Systems. In Proceedings of the Intelligent Transportation Systems, Vienna, Austria, 13-16 September 2003; pp. 590-597.

6. Zhang, F. The current situation and development thinking of the intelligent transportation system in China. In Proceedings of the International Conference on Mechanic Automation and Control Engineering, Wuhan, China, 26-28 June 2010; pp. 13-16.

7. Georga, E.; Protopappas, V.; Guille, A.; Fico, G.; Ardigo, D.; Arredondo, M.T.; Exarchos, T.P.; Polyzos, D.; Fotiadis, D.I. Data Mining for blood Glucose Prediction and Knowledge Discovery in Diabetic Patients: The METABO Diabetes Modeling and Management System. In Proceedings of the Annual International Conference of the IEEE EMBS, Minneapolis, MN, USA, 3-6 September 2009; pp. 5633-5636.

8. Kong, W.K.; Zhang, D.; Li, W. Palm print feature extraction using 2-D Gabor filters. Pattern Recognit. 2003, 36, 2339-2347. [CrossRef]

9. Jing, X.-Y.; Zhang, D.; Tang, Y.-Y. An improved LDA approach. IEEE Trans. Syst. Man Cybern. B Cybern. 2004, 34, 1942-1951. [CrossRef] [PubMed]

10. Bertozzi, M.; Broggi, A.; Fascioli, A. Vision-based intelligent vehicles: State of the art and perspective. Robot. Auton. Syst. 1998, 17, 56-63. [CrossRef]

11. Tsai, L.-W.; Hsieh, J.-W.; Fan, K.-C. Vehicle Detection Using Normalized Color and Edge Map. IEEE Trans. Image Process. 2007, 16, 850-854. [CrossRef] [PubMed]

12. Dubuisson, M.; Jain, A.; Taylor, W. A Vision-Based Vehicle Matching System. In Proceedings of the Intelligent Vehicles '94 Symposium, Paris, France, 24-26 October 1994; pp. 266-271.

13. Sotelo, M.A.; Nuevo, J.; Bergasa, L.M.; Ocana, M.; Parra, I.; Fernandez, D. Road Vehicle Recognition in Monocular Images. In Proceedings of the IEEE International Symposium on Strial Electronics, ISIE, Dubrovnik, Croatia, 20-23 June 2005; pp. 1471-1476.

14. Zhai, N.; Su, J.; Song, N.; Liu, Y.; Chen, Y. Research on Vehicle Type Recognition by Computer Vision Based on Invariant Moments. J. WUT Inf. Manag. Eng. 2007, 29, 7-10.

15. Yang, Z. Vehicle Type Recognition in Traffic Video Surveillance. Master's Thesis, Wuhan University of Technology, Wuhan, China, 2012.

16. Schlosser, C.; Reitberger, J.; Hinz, S. Automatic Car detection in high resolution urban scenes based on an adaptive $3 \mathrm{~d}$ model. In Proceedings of the IEEE Second GRSS/ISPRS Joint Workshop on Remote Sensing and Data Fusion over Urban Areas, Berlin, Germany, 22-23 May 2003; Volume 2, pp. 997-1000.

17. Low, D.G. Distinctive Image features from scale-invariant key-point. Int. J. Comput. Vis. 2004, 2, 91-110. [CrossRef]

18. Hagan, M.T.; Menhaj, M.B. Training Feed-forward networks with the marquardt algorithm. IEEE Trans. Neural Netw. 1994, 5, 295-301. [CrossRef] [PubMed]

19. Wang, Y. The Study and Design of Real-Time Image Processing Platform Based on FPGA. Master's Thesis, Northeastern University, Shenyang, China, 2011.

20. Cheung, S.C.; Kamath, C. Robust techniques for background subtraction in urban traffic video. Proc. Vis. Commun. Image Process. 2004, 5308, 881-892.

21. Kembhavi, A.; Harwood, D. Vehicle detection using partial least squares. IEEE Trans. Pattern Anal. Mach. Intell. 2011, 33, 1250-1265. [CrossRef] [PubMed]

22. Vedavathi, K.; Rap, K.S.; Devi, K.N. Unsupervised learning algorithm for time series using bivariate $\mathrm{AR}(1)$ model. Expert Syst. Appl. 2014, 41, 3402-3408. [CrossRef]

23. Wang, S.X.; Song, Z.-H.; Zhu, Z.-X.; Yang, B.-J.; Mao, E.-R.; Zhang, R. Study on crop image feature extraction of vehicle-based estimation system on large scale crop acreage. In Proceedings of the IEEE International Conference on Machine Learning and Cybernetics, Ningbo, China, 9-12 July 2007; pp. 377-382.

24. Huang, S.S.; Fu, L.C.; Hsiao, P.Y. Region-Level Motion-Based Background Modeling and Substraction Using MRFs. IEEE Trans. Image Process. 2007, 16, 1446-1456. [CrossRef] [PubMed]

25. $\mathrm{Hu}, \mathrm{M} . K$. Visual Pattern Recognition by Moment Invariants. IEEE Trans. Inf. Theory 1962, 8, 179-187. 
26. Kazemi, F.M.; Samadi, S.; Poorreza, H.R.; Akbarzadeh-T, M.R. Vehicle Recognition Using Curve let Transform and SVM. In Proceedings of the IEEE International Conference on Information Technology, Las Vegas, NV, USA, 2-4 April 2007; Volume 4, pp. 516-521.

27. Lin, S.-L.; Liu, Z. Parameter selection in SVM with RBF kernel function. J. Zhejiang Univ. Technol. 2007, 4, 163-167.

28. Jolly, D.; Lakshmanan, S.; Jain, K. Vehicle Segmentation and Classification Using Deformable Templates. IEEE Trans. Pattern Anal. Mach. Intell. 1996, 18, 293-308. [CrossRef]

29. Liu, S.-W.; Huang, H.-P.; Lin, C.-H.; Chien, I.L. Fuzzy-Logic-Based Supervisor of Insulin Bolus Delivery for Patients with Type 1 Diabetes Mellitus. Ind. Eng. Chem. Res. 2013, 52, 1678-1690. [CrossRef]

30. Wren, C.R.; Azarbayejani, A.; Darrell, T.; Pentland, A.P. Pfinder: Real-time tracking of the human body. IEEE Trans. PAMI 1997, 19, 780-785. [CrossRef]

31. Kijsirikul, B.; Ussivakul, N. Multiclass support vector machines using adaptive directed acyclic graph. In Proceedings of the International Joint Conference on Neural Networks, Honolulu, HI, USA, 12-17 May 2002; pp. 980-985.

32. Fei, B.; Liu, J.B. Binary tree of SVM: A new fast multiclass training and classification algorithm. IEEE Trans. Neural Netw. 2006, 17, 696-704. [CrossRef] [PubMed]

33. Rifkin, R.; Klautau, A. In defense of one-vs-all classification. J. Mach. Learn. Res. 2004, 5, 101-141.

34. Stauffer, C.; Grimson, W.E.L. Adaptive background mixture models for real-time tracking. In Proceedings of the IEEE Computer Society Conference on Computer Vision and Pattern Recognition, Fort Collins, CO, USA, 23-25 June 1999; IEEE Computer Society: Cambridge, MA, USA, 1999; pp. 1063-1069.

(C) 2017 by the authors. Licensee MDPI, Basel, Switzerland. This article is an open access article distributed under the terms and conditions of the Creative Commons Attribution (CC BY) license (http:/ / creativecommons.org/licenses/by/4.0/). 